\title{
Continuous declination system set up by observations of photoelectric astrolabe Mark I In Irkutsk
}

\author{
The first results of international cooperation between CSAO and VS NIIFTRI
}

\author{
Xu Jiayan ${ }^{1}$, Li Dongming ${ }^{3}$, Liu Jinmei ${ }^{1}$, Chen Hongqing ${ }^{1}$, Zhang Zhiwu ${ }^{1}$, S.I. Raschotin ${ }^{2}$, V.A. Emeliyanov ${ }^{2}$, \\ V.V. Kaplenko ${ }^{2}$, and V.T. Zalutsky ${ }^{2}$ \\ 1 Shaanxi Astronomical Observatory, Academia Sinica (CSAO), P.O. Box 18, Lintong, Xian 710600, PR China \\ 2 East-Siberean Research Institute for Physics Technical and Radio Engineering Measurements, Russia (VS NIIFTRI), 57, \\ Borodina Str., Irkutsk 664056, Russia \\ 3 Purple Mountain Observatory, Academia Sinica, Nanjing 210008, PR China
}

Received August 1; accepted November 10, 1997

\begin{abstract}
The Photoelectric Astrolabe Mark I (PHA I) has been revised with a new combined prism which could work as an almucantar with zenith distance of $45^{\circ}$, to measure continuous declinations at the latitude of Irkutsk, Russia $(\phi=52.2)$. The PHA I has been working at the astronomical base of VS NIIFTRI in Irkutsk since Nov. 1995 based on an international cooperation agreement of near 4 years for star catalogue and EOP measurements. The first observing program was ended in June 1997, giving corrections in both right ascension and declination to 200 stars with no blind zone in declination determination, which most astrolabe catalogues in the world usually would have ${ }^{1}$.
\end{abstract}

Key words: astrolabe - star catalogue

\section{Introduction}

The Chinese Photoelectric Astrolabe Mark I (PHA I) was installed at Shaanxi Astronomical Observatory in 1971, and started to work in 1972. The PHA I worked at almucantar with zenith distance of $30^{\circ}$ before the $1990 \mathrm{~s}$, mainly for Earth Orientation Parameters (EOP) measurements, but partly for star catalogues. Since French astronomers (Guinot et al. 1961) published their first creative results of 571 star positions observed with the Danjon astrolabe, many astrolabe catalogues have been published, and have

Send offprint requests to: Xu Jiayan

1 Appendix is only available in electronic form at the CDS via anonymous ftp to cdsarc.u-strasbg.fr (130.79.128.5) or via http://cdsweb.u-strasbg.fr/Abstract.html played quite good role for compilation of the FK5 system. Most of those catalogues have blind zones with declination width of about 25 degrees. As most astrolabes in the world, the PHA I also had a blind zone in declination determination. In order to do more and better work for star catalogues, and to eliminate the blind zone in declination measurements, both CSAO and VS NIIFTRI had signed a scientific cooperation agreement about jointly using the PHA I in Irkutsk, Russia for star catalogue and EOP measurements in Sep. 1993. To do this, CSAO revised their PHA I with a new combined prism which could work on almucantar with zenith distance of $45^{\circ}$ in 1993-94. According to the agreement, the PHA I was installed at the astronomical base of VS NIIFTRI in Irkutsk in Oct. 1995, and started to work in Nov. 1995.

\section{The program and the instrument}

The first observing program involving 668 star transits contains 12 groups. Since the program was used for both EOP measurements and a star catalogue, it contains 496 basic stars and 172 catalogue stars (both from FK5 and FK5supp.). The program was observed for 19 months, and 329 clear nights, with 1146 groups in total. Some winter groups were observed 132 times at the maximum, and summer groups only 57 times at the minimum.

The PHA I is a fully-automatic astrolabe, controlled by an IBM PC 286 computer which could also be used for photon-counting data reduction and data solution after observation. The PHA I is equipped with two photoncounting detectors which could reach faint stars to mag. 9.5 with an equivalent aperture of $75 \mathrm{~mm}$ for each pupil of two images (the primary mirror aperture of the PHA $\mathrm{I}$ is $150 \mathrm{~mm}$ ). It has an inductosyn with precision of $3^{\prime \prime}$ 
Table 1. Observations in each month

\begin{tabular}{|c|c|c|c|c|c|c|}
\hline Mon & $\begin{array}{l}\text { Number } \\
\text { of } \mathrm{Gr}\end{array}$ & $\begin{array}{l}M_{\mathrm{rms}} \\
\left({ }^{\prime \prime}\right)\end{array}$ & $\begin{array}{l}E_{\text {uto }} \\
\text { (s) }\end{array}$ & $\begin{array}{c}M_{\text {temp }} \\
\left({ }^{\circ} \mathrm{C}\right)\end{array}$ & $\begin{array}{c}M_{\text {pres }} \\
(\mathrm{mb})\end{array}$ & $\begin{array}{c}M_{\mathrm{dz}} \\
\left({ }^{\prime \prime}\right)\end{array}$ \\
\hline 1 & 167 & \pm 0.246 & \pm 0.0053 & -19.1 & 958.0 & 1.883 \\
\hline 2 & 203 & 0.236 & 0.0051 & -18.4 & 963.9 & 1.832 \\
\hline 3 & 165 & 0.225 & 0.0049 & -8.7 & 958.2 & 1.840 \\
\hline 4 & 99 & 0.223 & 0.0050 & -0.3 & 956.0 & 1.798 \\
\hline 5 & 40 & 0.211 & 0.0044 & 6.1 & 952.0 & 1.815 \\
\hline 6 & 55 & 0.226 & 0.0051 & 9.7 & 945.3 & 1.714 \\
\hline 7 & 49 & 0.215 & 0.0046 & 14.5 & 945.9 & 1.788 \\
\hline 8 & 46 & 0.214 & 0.0044 & 12.3 & 947.6 & 1.768 \\
\hline 9 & 31 & 0.220 & 0.0045 & 3.1 & 952.4 & 1.715 \\
\hline 10 & 58 & 0.213 & 0.0046 & -2.2 & 954.7 & 1.736 \\
\hline 11 & 107 & 0.224 & 0.0047 & -11.4 & 959.3 & 2.013 \\
\hline 12 & 126 & 0.247 & 0.0052 & -15.2 & 958.7 & 1.977 \\
\hline & \multicolumn{4}{|c|}{ mean } & & \\
\hline & 1146 & 0.230 & & -8.3 & & \\
\hline
\end{tabular}

Table 2. Group differences

\begin{tabular}{crrrrrr}
\hline $\begin{array}{c}G_{i+1} \\
-G_{i}\end{array}$ & \multicolumn{1}{c}{$\begin{array}{c}\mathrm{du} 0 \\
(\mathrm{~s})\end{array}$} & \multicolumn{1}{c}{$\begin{array}{c}\mathrm{dy} \\
\left({ }^{\prime \prime}\right)\end{array}$} & \multicolumn{1}{c}{$\begin{array}{c}\mathrm{ddz} \\
\left({ }^{\prime \prime}\right)\end{array}$} & $\begin{array}{c}E_{\mathrm{duo}} \\
(\mathrm{s})\end{array}$ & \multicolumn{1}{c}{$\begin{array}{c}E_{\mathrm{dy}} \\
\left({ }^{\prime \prime}\right)\end{array}$} & $\begin{array}{c}E_{\mathrm{ddz}} \\
\left({ }^{\prime \prime}\right)\end{array}$ \\
\hline $2-1$ & 0.0105 & -0.026 & 0.033 & \pm 0.0009 & \pm 0.012 & \pm 0.007 \\
$3-2$ & 0.0009 & 0.002 & 0.028 & 0.0009 & 0.011 & 0.007 \\
$4-3$ & -0.0004 & 0.034 & 0.023 & 0.0008 & 0.010 & 0.006 \\
$5-4$ & -0.0019 & -0.156 & -0.008 & 0.0009 & 0.012 & 0.007 \\
$6-5$ & -0.0026 & 0.045 & 0.034 & 0.0008 & 0.011 & 0.006 \\
$7-6$ & -0.0003 & -0.045 & -0.001 & 0.0008 & 0.010 & 0.006 \\
$8-7$ & 0.0026 & 0.007 & -0.014 & 0.0008 & 0.010 & 0.006 \\
$9-8$ & 0.0024 & 0.072 & 0.057 & 0.0013 & 0.016 & 0.009 \\
$10-9$ & 0.0073 & 0.058 & -0.018 & 0.0020 & 0.021 & 0.012 \\
$11-10$ & -0.0066 & 0.013 & 0.017 & 0.0017 & 0.018 & 0.010 \\
$12-11$ & 0.0071 & -0.140 & 0.045 & 0.0014 & 0.015 & 0.009 \\
$1-12$ & -0.0057 & 0.106 & 0.022 & 0.0012 & 0.016 & 0.008 \\
closing & & & & & & \\
error, $k$ & 0.0131 & -0.029 & 0.217 & 0.0041 & 0.049 & 0.027 \\
\hline
\end{tabular}

in azimuth positioning. The PHA I could work for more than 12 hours in winter at temperatures down to $-35^{\circ} \mathrm{C}$ with some electronic parts being heated partly. Since the combined prism is made of zerodur, the zenith distance of the almucantar is very stable either over a year, or during a night (see Tables 1 and 2). This feature benefits not only its observing precision, but declination measurements as well, making the closing error $k$ of zenith distance (being $0.217^{\prime \prime}$ for 12 groups) much smaller than that of the Danjon astrolabe. The mean RMS of 1146 groups observed in 329 nights with mean temperature of $-8.3^{\circ} \mathrm{C}$ is $\pm 0.230^{\prime \prime}$ which is equivalent to $\pm 0.190^{\prime \prime}$ of RMS for zenith distance of $30^{\circ}$. This is excellent precision under quite low temperature.

Since the data of astronomical time and latitude measured by the PHA I are very good in both precision and accuracy, the PHA I had quite large weights in the Russian EOP service system among about 20 optical instruments in the year 1996. Besides this, the data also have been used to get corrections in both right ascension and declination for 200 stars, which are listed in order of declination in the appendix.

\section{Treatment of systematic errors}

As shown in the case of the visual Danjon astrolabe (Guinot 1958), the PHA I is not exempt of a spectral equa-
Table 3. Secondary corrections for spectrum and residuals

\begin{tabular}{crrr}
\hline Spect & Num & $\begin{array}{c}\text { Corr } \\
\left({ }^{\prime \prime}\right)\end{array}$ & \multicolumn{1}{c}{$\begin{array}{c}\text { Resid } \\
\left({ }^{\prime \prime}\right)\end{array}$} \\
\hline O-B & 79 & 0.000 & 0.002 \\
A & 117 & -0.030 & 0.000 \\
F & 78 & -0.012 & 0.004 \\
G & 80 & 0.005 & -0.005 \\
K & 144 & 0.020 & 0.000 \\
M & 65 & 0.030 & -0.001 \\
\hline
\end{tabular}

Table 4. Mean residual with magnitudes

\begin{tabular}{crrr}
\hline$M_{\text {mag }}$ & $N_{\text {um }}$ & \multicolumn{1}{c}{$\begin{array}{c}M_{v} \\
\left({ }^{\prime \prime}\right)\end{array}$} & \multicolumn{1}{c}{$\begin{array}{c}E_{\mathrm{mv}} \\
\left({ }^{\prime \prime}\right)\end{array}$} \\
\hline 3.1 & 50 & -0.004 & \pm 0.171 \\
4.1 & 83 & 0.008 & 0.168 \\
5.0 & 107 & 0.013 & 0.172 \\
5.7 & 164 & -0.011 & 0.173 \\
6.4 & 77 & 0.002 & 0.170 \\
7.1 & 44 & 0.012 & 0.172 \\
8.4 & 38 & -0.018 & 0.193 \\
\hline
\end{tabular}

tion. For the PHA I, two photomultiplier tubes with cathodes of S-20 type are used to detect two images of starlight respectively, and multiple media layers with high reflection $(>98 \%)$ and bandwidth of $260 \mathrm{~nm}$ (from 380 to $640 \mathrm{~nm}$ ) were put on the surfaces of the combined prism. Each star for different spectral types has its own effective wavelength which causes different atmospheric refractions, so-called spectral equations (errors). The corrections for spectral equations at zenith distance $30^{\circ}$ could reach $-0.15^{\prime \prime}$ for early-type stars (O, B types), and $0.15^{\prime \prime}$ for late-type stars (K, M types) (Yang Tinggao et al. 1980). These are much larger than those for the Danjon astrolabe. The first order of spectral corrections used here is derived from the values obtained earlier at zenith distance $30^{\circ}$, but multiplied by a factor of $1.732\left(\tan 45^{\circ} / \tan 30^{\circ}\right)$. The second order of spectral corrections was found from analysis of residuals of stars with different spectral types, shown in Table 3. After having used the second-order corrections, spectral equations could be eliminated quite well, better than $0.01^{\prime \prime}$ (see residuals in Table 3). Table 4 does not provide magnitude equations, but does show that the observing precision is slightly lower for faint stars (around magnitude 9).

\section{Method of data reduction}

The Fifth Fundamental Catalogue (FK5) and the IAU (1976) System of Astronomical Constants have been used in the data reduction to obtain the residuals.

As for previous reductions (see for instance Guinot 1958), the chain method was used to get group corrections in this work. Since winter groups have been observed more 
Table 5. Group corrections

\begin{tabular}{rrrrrrr}
\hline Group & \multicolumn{1}{c}{$\begin{array}{c}C_{\mathrm{u}} \\
(\mathrm{s})\end{array}$} & \multicolumn{1}{c}{$\begin{array}{c}C_{\mathrm{y}} \\
\left({ }^{\prime}\right)\end{array}$} & \multicolumn{1}{c}{$\begin{array}{c}C_{\mathrm{dz}} \\
\left({ }^{\prime \prime}\right)\end{array}$} & \multicolumn{1}{c}{$\begin{array}{c}E_{\mathrm{cu}} \\
(\mathrm{s})\end{array}$} & \multicolumn{1}{c}{$\begin{array}{c}E_{\mathrm{cy}} \\
\left({ }^{\prime \prime}\right)\end{array}$} & $\begin{array}{c}E_{\mathrm{cdz}} \\
\left({ }^{\prime \prime}\right)\end{array}$ \\
\hline 1 & 0.0059 & -0.054 & 0.021 & \pm 0.0010 & \pm 0.013 & \pm 0.007 \\
2 & -0.0039 & -0.030 & 0.001 & 0.0010 & 0.011 & 0.007 \\
3 & -0.0041 & -0.034 & -0.014 & 0.0008 & 0.010 & 0.006 \\
4 & -0.0032 & -0.069 & -0.027 & 0.0009 & 0.012 & 0.006 \\
5 & -0.0008 & 0.085 & -0.006 & 0.0008 & 0.011 & 0.006 \\
6 & 0.0023 & 0.038 & -0.031 & 0.0008 & 0.010 & 0.006 \\
7 & 0.0031 & 0.082 & -0.020 & 0.0009 & 0.011 & 0.006 \\
8 & 0.0010 & 0.074 & 0.005 & 0.0015 & 0.018 & 0.009 \\
9 & 0.0000 & -0.001 & -0.030 & 0.0022 & 0.023 & 0.013 \\
10 & -0.0041 & -0.065 & 0.034 & 0.0017 & 0.018 & 0.010 \\
11 & 0.0046 & -0.082 & 0.044 & 0.0014 & 0.016 & 0.009 \\
12 & -0.0010 & 0.055 & 0.023 & 0.0013 & 0.016 & 0.009 \\
\hline
\end{tabular}

times, and summer groups less, we use precise formulae to calculate group corrections (Thomas 1966):

$C[i]=-\frac{1}{n} \sum_{r=0}^{n-1}(r-F[i]) \triangle x[i+r]$.

Here $C[i]$ is correction of group $i(i=1, \ldots, n ; n=12)$, $\triangle x[i+r]=x[i+r+1]-x[i+r]$, and

$F[i]=\sum_{r=0}^{n-1} r E^{2}[i+r] / E_{k}^{2}$.

Here $E[i+r]$ is the mean error of group difference $\triangle x[i+r]$ and $E_{k}$ the error of closing error $k$. The error of group correction $C[i]$ is given by:

$E c[i]= \pm \frac{1}{n}\left\{\sum_{r=0}^{n-1}(r-F[i])^{2} E^{2}[i+r]\right\}^{\frac{1}{2}}$.

The mean residual of star $j$ in group $i$ with respect to the mean group is:

$M[j]=V[j]+C u[i] 15 \cos \phi \sin A[j]+C y[i] \cos A[j]+C z[i] .(4)$

Here $V[j]$ is the average of residuals of $\operatorname{star} j$ in group $i$.

Through observing double transits of a star (one in the East of almucantar, the other in the West), we could have two residuals $M_{\mathrm{e}}$ and $M_{\mathrm{w}}$ which could give corrections to both right ascension and declination of the star (Guinot 1958):

$\triangle \alpha=\frac{M_{\mathrm{e}}-M_{\mathrm{w}}}{30|\sin A| \cos \phi}$,

$\triangle \delta=-\frac{M_{\mathrm{e}}+M_{\mathrm{w}}}{2 \cos Q}$.

Here $\phi$ is the latitude of the instrument position.

\section{Precision of corrections}

Precision of corrections in right ascension depends largely on the azimuth of the star observed, and precision of corrections in declination depends on $\cos Q$. For most astrolabes and middle latitudes, we often have $\cos Q=0$ or else $\cos Q$ stays between -0.35 and +0.35 for many stars that have either no corrections or no precise corrections in declination. Those stars occupy quite wide zones in declination, which is often called the blind zone in declination measurements. But the PHA I in Irkutsk always has

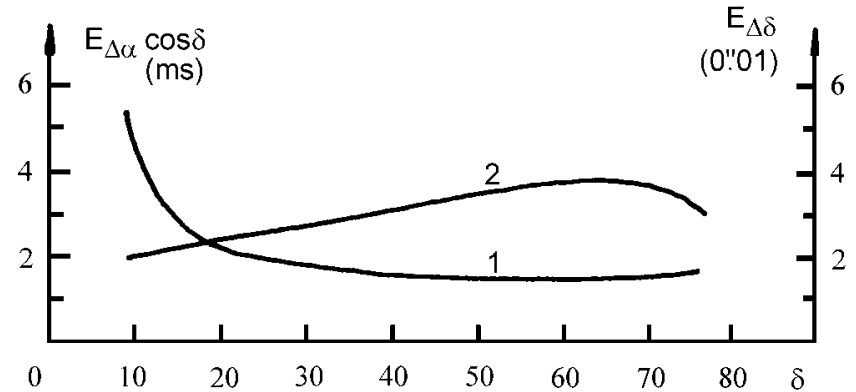

Fig. 1. Precision varies with declination for $\phi=52^{\circ} .2, Z=45^{\circ}$

$\cos Q \geq 0.5$, so it has no such blind zone (Li Dongming et al. 1983). To see this, we could use another expression of $\cos Q$ (Xu Jiayan et al. 1994):

$\cos Q=(\sin \phi / \cos Z-\sin \delta) / \tan Z / \cos \delta$.

When

$\phi+Z>90^{\circ}$,

we always have $\cos Q>0$. Since for the PHA I, $Z=45^{\circ}$, $\phi=52^{\circ} .2, \phi+Z=97^{\circ} .2$, we always have $\cos Q \geq 0.5$. This is how we eliminate the blind zone in declination determination.

We calculated precisions of corrections $\triangle \alpha \cos \delta$ and $\triangle \delta$ theoretically. In this calculation, we use mean RMS of $0.23^{\prime \prime}$ and the assumption of observing each star 70 times for $\phi=52.2$ at different declinations. The results are shown in Fig. 1, where curve 1 stands for $E_{\triangle \alpha} \cos \delta$ varying with $\delta$, and curve 2 stands for $E_{\triangle \delta}$ varying with $\delta$. The trend of $E_{\triangle \alpha} \cos \delta$ and $E_{\triangle \delta}$ varying with declination in the appendix are in good accordance with the two curves in Fig. 1.

\section{Results}

From the observations of program 1 used from 03 Nov. 1995 to 25 June 1997, we get corrections in both coordinates for 200 stars. Coverage of declination is about 65 degrees (from 9.5 to 74.5) with no gap. The results are given in the appendix, in the order of declinations from $9^{\circ}$ to $75^{\circ}$. One sees from the list that the formal errors $E_{\triangle \alpha}$ vary with $\sin A$. The formal errors $E_{\triangle \delta}$ vary with $\delta(\cos Q)$. When $\delta=63^{\circ}, \cos Q$ reachs its minimum (0.5) and the $E_{\triangle \delta}$ reach maximum. For $E_{\triangle \alpha} \cos \delta, 93 \%$ are better than $\pm 4 \mathrm{~ms}, 85 \%$ better than $\pm 3 \mathrm{~ms}$, and their mean value is $\pm 2.3 \mathrm{~ms}$. For $E_{\triangle \delta}, 86 \%$ are better than $\pm 0.040^{\prime \prime}, 51 \%$ better than $\pm 0.030^{\prime \prime}$, and their mean value is $\pm 0.031^{\prime \prime}$. If we had observed more nights in the summer of 1996, we would have got better precisions for some stars in both $\triangle \alpha$ and $\triangle \delta$. One can see from Table 5 that the precisions of group corrections for numbers 9 and 10 are about 2 times worse than the others, which makes mean residuals $M$ for some stars in the groups 9 and 10 less precise. 


\section{Conclusion and perspectives}

As the conclusion, we should point out the fact that, using the high latitude position of the station within the cooperation between CSAO and VS NIIFTRIand the optical square with an angle of $45^{\circ}$, this catalogue is the first one without any blind zone and has a coverage of $65^{\circ}$. The detectors can reach magnitude 9.5 , and observations could be made down to temperature $-35^{\circ} \mathrm{C}$, which is an improvement regarding other similar instruments.

Even though the Hipparcos catalogue comes into use, observing some bright stars well-chosen by ground-based instrumentations repeatedly is still useful for maintenance of the Hipparcos reference system and for improvement of the Hipparcos proper motion system. On the other hand, observing some radio stars is helpful for connecting the Hipparcos reference system to the radio reference system.

Acknowledgements. The authors would like to give many thanks to the China National Natural Science Foundation, to the Bureau of Basic Research and Bureau of International Cooperation of the Chinese Academy of Sciences, and the China Astronomical Committee, which give our project great support in every step of this cooperation. We also offer many thanks to the Russian Ministry of Science and Technology Policy, which gives the cooperation strong support.

\section{References}

Guinot B., 1958, Bull. Astrn. t22, f1

Guinot B., et al., 1961, Bull. Astrn. 23, 307

Li Dongming, Xu Jiayan, Luo Dingjiang, 1983, Method of Equal Altitude And Its Application In Fundamental Astrometry

Thomas D.V., 1966, Roy. Astron. Soc. Bull. 127, 129

$\mathrm{Xu}$ Jiayan, Wang Zhengming, 1994, Chinese Astron. Astrophys. 18, 462

Yang Tinggao, Xu Jiayan, 1980, Acta Astron. Sin. 21, 73 